\title{
Negative pressure wound therapy (NPWT) treatment of total supra-aortic debranching graft infection
}

Raffaello Bellosta, Luca Luzzani, Francesca Bontempi, Monica Vescovi, Antonio Sarcina

Vascular Surgery Unit, Cardiovascular Surgery Department, Poliambulanza Foundation Hospital, Brescia, Italy

Submitted: 23 July 2015

Accepted: 17 September 2015

Arch Med Sci 2018; 14, 2: 466-469

DOI: 10.5114/aoms.2016.59603

Copyright $\odot 2016$ Termedia \& Banach

Endovascular treatment strategies for aortic arch disease have been increasingly prevalent over the last decade as a less invasive option for patients not eligible for conventional open repair, a procedure that is still associated with high complication and mortality rates [1-3].

Anatomical and pathological aortic arch peculiarities often require a hybrid approach to achieve an adequate proximal landing zone and preserve cerebral and spinal cord perfusion [4]. Various surgical options have been developed for revascularization of supra-aortic arteries, including partial or complete artery debranching with bypass reconstruction or transposition. Although these hybrid procedures represent an effective alternative for high risk patients, they are also associated with significant cardiac, neurological (stroke, intracerebral hemorrhage or spinal cord ischemia), and pulmonary renal complication rates [5, 6]. Endovascular stent graft infection is a rare complication of endovascular treatment. Among the many possible complications, supra-aortic graft infection in hybrid aortic arch repair has not been reported in the literature yet.

Patient consent to publish the data was obtained.

An Institutional Review Board (IRB) does not exist at our institution. The principles of the Declaration of Helsinki were followed.

A 75-year-old man presented with an asymptomatic $74 \mathrm{~mm}$ diameter aortic arch aneurysm; associated comorbidities were hypertension, obesity, chronic obstructive pulmonary disease and atrial fibrillation. Six years previously, the patient underwent open repair with an aorto-biiliac Dacron graft for an abdominal aortic aneurysm. Because of these comorbidities, a hybrid approach (open and endovascular) was chosen. A computed tomography (CT) scan showed a large arch aneurysm (74 mm diameter) involving the left subclavian artery (Figure 1).

To obtain an adequate landing zone (zone 0 , according to the Ishimaru classification [7]) total supra-aortic trunk debranching was planned.

Through a median sternotomy, the ascending aorta and supra-aortic trunk were isolated. A side clamp was applied to the distal ascending aorta and an end-to-side anastomosis with a $10 \mathrm{~mm}$ Dacron graft was performed (C.R. Bard, Inc., Murray Hill, New Jersey, USA). A $7 \mathrm{~mm}$ and $8 \mathrm{~mm}$ trifurcated Dacron graft was tailored manually. Distal end-to-end anastomoses were then sequentially performed on the anonymous artery and the left common carotid artery. Revascularization of the left subclavian artery required a supra-clavicular cervicotomic access to perform an end-to-side anastomosis associated with pre-vertebral artery ligation. Near infrared spectroscopy was used to monitor cerebral perfusion during carotid revascularization.

\author{
Corresponding author: \\ Raffaello Bellosta MD \\ Vascular Surgery Unit \\ Cardiovascular Surgery \\ Department \\ Poliambulanza Foundation \\ Hospital \\ 57 Via Bissolati St \\ 25100 Brescia, Italy \\ Phone: +390303518255 \\ E-mail: raffaello.bellosta@ \\ poliambulanza.it
}




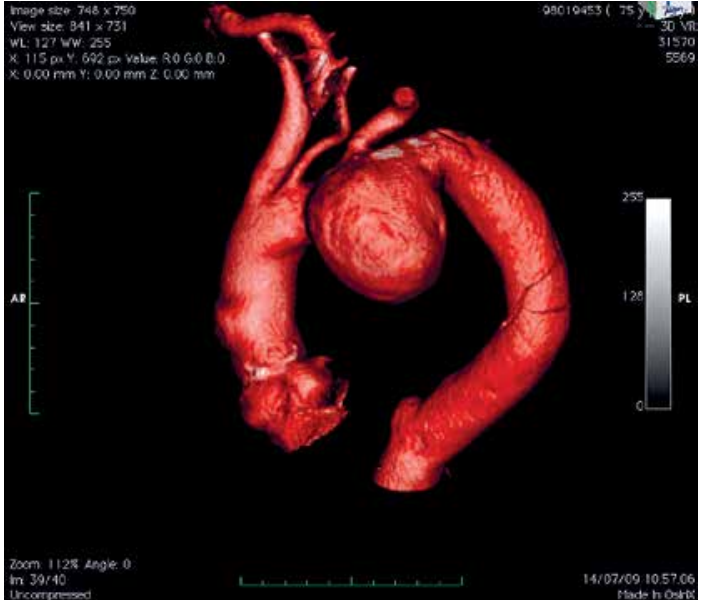

Figure 1. Computed tomography scan showing a large (74 $\mathrm{mm}$ diameter) aortic arch aneurysm

Following the open thoracic procedure, the left common femoral artery was surgically prepared. Regular graft patency with complete visualization of supra-aortic branches and a bulky arch aneurysm were confirmed by aortic angiography. A 24 Fr Gore sheath (W. L. Gore Corp, Flagstaff, AZ) was introduced and the first stent graft (Gore TAG 3715) was deployed in the descending aorta. This was then extended proximally with a second stent graft (TAG 4015) into the aortic arch. Complete aneurysm exclusion without evidence of endoleaks was confirmed by final angiographic control. The total procedure time was $240 \mathrm{~min}$.

Antibiotic prophylaxis with cefazolin was administered preoperatively and continued until postoperative day 4 . The postoperative course was uneventful. Aneurysm exclusion with no endoleaks and graft patency was confirmed by a CT scan performed 10 days following the implantation. The patient was discharged on the $13^{\text {th }}$ post-operative day with no local or systemic complications.
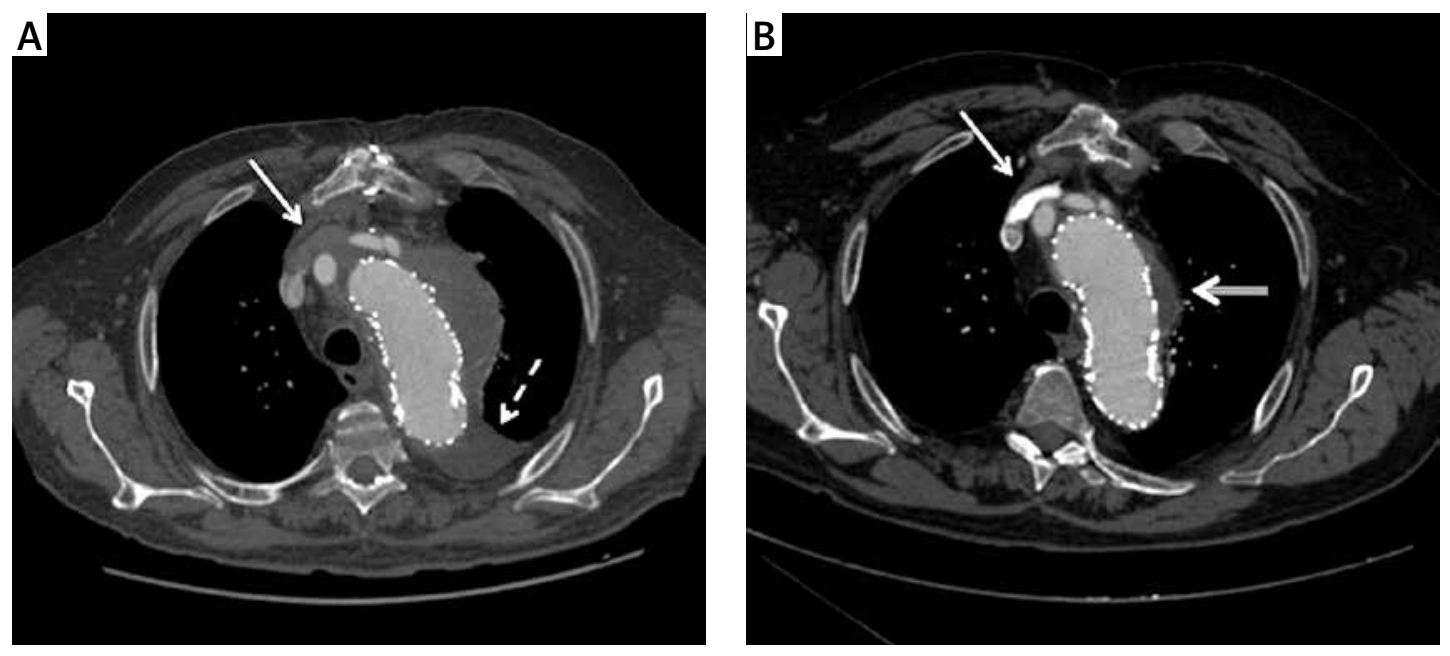

Figure 3. Computed tomography scan before (A) and after (B) treatment. A - Large fluid collection enveloping anonymous vein and Dacron graft (continuous arrow) with left pleural effusion (dotted arrow). B - Resolution of infectious process (continuous arrow) with shrinkage of the aneurysm (bold arrow) 
The negative pressure wound therapy (NPWT) device was removed following confirmation that the patient was free from further secretion and that pathogenic bacterial growth was absent in a negative culture sample. Negative pressure wound therapy therapy lasted 30 days.

After discharge, the patient received doxycycline twice daily and oxygen therapy in a decompression chamber at 1 month to complete the wound healing.

At 36-month follow-up, the patient was completely asymptomatic, with no reoccurring wound dehiscence; a second CT scan confirmed complete resolution of the infectious process, regular patency of the supra-aortic grafts and shrinkage of the aneurysm (Figure $3 \mathrm{~B}$ ).

Hybrid repair of the aortic arch with supra-aortic debranching prior to stent graft deployment provides the advantage of reducing invasiveness by avoiding aortic cross-clamping and circulatory arrest.

Up to $40 \%$ of thoracic aneurysms may require coverage of one or more of the supra-aortic trunks and, therefore, open debranching may be used to achieve an adequate proximal landing zone in subsequent endovascular procedures. The accessibility of hybrid procedures expands the number of patients eligible for endovascular repair, thus avoiding more invasive treatment in high-risk patients. In open vascular surgery, graft infection is a complication with high mortality. However, graft infections related to aortic arch debranching have not been reported in the literature yet. It remains controversial whether synchronous or staged open and endovascular treatment is preferable in hybrid procedures [8]. Some authors suggest a staged approach (open first, endovascular second stage), which provides time to evaluate the neurological status before the endovascular procedure and reduces the operation time [9]. Others suggest that any procedural problem occurring during intervention, either endovascular or surgical, may be better addressed using a single stage procedure [5]. However, the longer operation time may increase the risk of renal and cardiac complications.

Simultaneous procedures could expose the patient to a higher risk of graft infection because of the procedure length and the involvement of various physicians (i.e., cardiac surgeon, vascular surgeon, radiologist). Nevertheless, more cases should be recorded and reviewed to define a significant correlation.

There is no definitive therapy for graft infections to date. Once the thoracic graft infection is discovered, the treatment consists of a regimen of high-dose intravenous antibiotics, complete debridement of infected and necrotic tissue surrounding the prosthetic material, mediastinal ir- rigation, in situ replacement of the infected aortic graft with a new graft, and graft coverage with viable tissue [10].

Negative pressure wound therapy was previously described for the treatment of osteomyelitis and originally applied to mediastinal infection. Its application has been steadily expanding and presently is the treatment of choice in several institutes for post-sternotomy mediastinitis

Saiki et al. [11] successfully treated 5 patients with thoracic aortic graft infections using the NPWT system but not in debranching revascularization (ascending aorta and aortic root).

Graft infections are treated by extensive debridement and removal of all infected tissue, before positioning NPWT therapy.

Negative pressure wound therapy, which was introduced as a commercial product $(\mathrm{KCl}$ USA, Inc., San Antonio, TX) less than 20 years ago, has revolutionized the treatment of complex wounds. Indicated for a wide variety of wound types, NPWT is an adjunctive therapy that can be used safely in a range of care settings. Therefore, we recommend the use of the vacuum therapy system in patients with prosthetic graft infection.

\section{Conflict of interest}

The authors declare no conflict of interest.

\section{References}

1. Kpodonu J, Diethrich EB. Hybrid interventions for the treatment of the complex aortic arch. Perspect Vasc Surg Endovasc Ther 2007; 19: 174e84.

2. Antoniou GA, El Sakka K, Hamady M, Wolfe JH. Hybrid treatment of complex aortic arch disease with supra-aortic debranching and endovascular stent graft repair. Eur J Vasc Endovasc Surg 2010; 39: 683-90.

3. Kazui T, Yamashita K, Washiyama N, et al. Aortic arch replacement using selective cerebral perfusion. Ann Thorac Surg 2007; 83: S796-8; discussion: S824-31.

4. Schumacher H, Bockler D, Bardenheuer H, Hansmann J, Allenberg JR. Endovascular aortic arch reconstruction with supra-aortic transposition for symptomatic contained rupture and dissection: early experience in 8 highrisk patients. J Endovasc Ther 2003; 10: 1066-74.

5. Younes HK, Davies MG, Bismuth J, et al. Hybrid thoracic endovascular aortic repair: pushing the envelope. J Vasc Surg 2010; 51: 259-66.

6. Chiesa R, Melissano G, Tshomba Y, et al. Ten years of endovascular aortic arch repair. J Endovasc Ther 2010; 17: 1-11.

7. Mitchell RS, Ishimaru S, Ehrlich MP, et al. First International Summit on Thoracic Aortic Endografting: roundtable on thoracic aortic dissection as an indication for endografting. J Endovasc Ther 2002; 9 Suppl 2: ॥198-105.

8. Koullias GJ, Wheatley GH $3^{\text {rd }}$. State-of-the-art of hybrid procedures for the aortic arch: a meta-analysis. Ann Thorac Surg 2010; 90: 689-97.

9. Geisbüsch P, Kotelis D, Müller-Eschner M, Hyhlik-Dürr A, Böckler D. Complications after aortic arch hybrid repair. J Vasc Surg 2011; 53: 935-41. 
10. Chan YC, Cheng SW, Ting AC, Ho P. Supra-aortic hybrid endovascular procedures for complex thoracic aortic disease: single center early to midterm results. J Vasc Surg 2008; 48: 571-9.

11. Saiki Y, Kawamoto S, Sai S, Tabayashi K. An effective vacuum-assisted closure treatment for mediastinitis with aortic arch replacement. Interact Cardiovasc Thorac Surg 2008; 7: 712-4. 